
\title{
R Research Square \\ Resonant Collisions Among Two-Dimensional Localized Waves in the Mel'nikov Equation
}

\section{Yinshen Xu}

Institute for Advanced Study: Central European University Institute for Advanced Study

\section{Dumitru Mihalache}

Horia Hulubei National Institute of Physics and Nuclear Engineering Nuclear Physics Department \& Tandem Accelerator Department

Jingsong He ( $\nabla$ hejingsong@szu.edu.cn )

Institute for advanced Study, shenzhen university

\section{Research Article}

Keywords: Two-dimensional doubly localized rogue waves, Resonant collision, Mel'nikov equation

Posted Date: August 30th, 2021

DOl: https://doi.org/10.21203/rs.3.rs-819414/v1

License: (c) (i) This work is licensed under a Creative Commons Attribution 4.0 International License.

Read Full License

Version of Record: A version of this preprint was published at Nonlinear Dynamics on October 4th, 2021. See the published version at https://doi.org/10.1007/s11071-021-06880-8. 


\title{
Resonant collisions among two-dimensional localized waves in the Mel'nikov equation
}

\author{
Yinshen Xu • Dumitru Mihalache • \\ Jingsong $\mathrm{He}^{\dagger}$
}

Received: date / Accepted: date

\begin{abstract}
We study the resonant collisions among different types of localized solitary waves in the Mel'nikov equation, which are described by exact solutions constructed using Hirota's direct method . The elastic collisions among different solitary waves can be transformed into resonant collisions when the phase shifts of these solitary waves tend to infinity . First, we study the resonant collision among a breather and a dark line soliton. We obtain two collision scenarios: (i) the breather is semi-localized in space and is not localized in time when it obliquely intersects with the dark line soliton, and (ii) the breather is semi-localized in time and is not localized in space when it parallelly intersects with the dark line soliton. The resonant collision of a lump and a dark line soliton, as the limit case of resonant collision of a breather and a dark line soliton, shows the fusing process of the lump into the dark line soliton. Then we investigate the resonant collision among a breather and two dark line solitons. In this evolution process we also obtain two dynamical behaviors: (iii) when the breather and the two dark line solitons obliquely intersect each other we get that the breather is completely localized in space and is not localized in time, and (iv) when the breather and the two dark line solitons are parallel to each other, we get that the breather is completely localized in time and is not localized in space. The resonant collision of a lump and two dark line solitons is obtained as the limit case of the resonant collision among a breather and two dark line solitons. In this special case the lump first detaches from a dark line soliton and then disappears into the other dark line soliton. Eventually, we
\end{abstract}

Corresponding author email: hejingsong@szu.edu.cn

Yinshen $\mathrm{Xu}$

Institute for Advanced Study, Shenzhen University, Shenzhen, Guangdong 518060, P. R. China

Dumitru Mihalache

Horia Hulubei National Institute for Physics and Nuclear Engineering, P.O. Box MG-6, Magurele, RO 077125, Romania

Jingsong $\mathrm{He}$

Institute for Advanced Study, Shenzhen University, Shenzhen, Guangdong 518060, P. R. China 
also investigate the intriguing phenomenon that when a resonant collision among a breather and four dark line solitons occurs, we get the interesting situation that two of the four dark line solitons are degenerate and the corresponding solution displays the same shape as that of the resonant collision among a breather and two dark line solitons, except for the phase shifts of the solitons, which are not only dependent of the parameters controlling the waveforms of the solitons and the breather, but also dependent of some parameters irrelevant to the waveforms.

Keywords Two-dimensional doubly localized rogue waves · Resonant collision · Mel'nikov equation

\section{Introduction}

The theoretical and experimental studies of nonlinear dynamics and unique interaction scenarios of various types of nonlinear waveforms are flourishing due to diverse applications in physics and engineering. The rather complicated evolution of the nonlinear wavepackets are described by a plethora of nonlinear partial differential equations, which have been comprehensively studied both analytically and numerically, during the past several decades. Zabusky and Kruskal published in 1965 [1] a pioneering work that put forward for the first time the idea that the Korteweg-de Vries (KdV) equation could admit soliton solutions. Shortly afterwards, a plenty of methods to solve and analyze the solutions of various nonlinear partial differential equations, such as the inverse scattering method [2] and the Hirota method [3], came out into play. These powerful methods have enabled researchers to analytically solve the nonlinear partial differential equations more efficiently, so in 1974, the $N$-soliton solutions of the $\mathrm{KdV}$ equation were found in Ref.[4] by using the inverse scattering method. A few years later, rational solutions of the $\mathrm{KdV}$ equation have been obtained by Airault et al. [5], and other researchers deeply explored the solitary and rational solutions of nonlinear evolution equations [6,7]. Satsuma and Ablowitz investigated the rational solutions of the Kadomtsev-Petviashvili (KP) equation and of the two-dimensional nonlinear Schrödinger (NLS) equation in 1979 [8].

The interacting property of the above-mentioned solutions is one of long-term topics based on the explicit forms in order to confirm the particle-like behavior and to find some unique characters of nonlinear waves. After the pioneering studies on resonantly interacting solitary waves, which were published in 1977 by Miles [9] and by Newell et al. [10], these investigations gradually prompted the attention of scientists working in the area of nonlinear dynamics. In 1980, Kako and Yajima [11] studied the resonant solitary wave solution of the Boussinesq-like ion-acoustic equation in two-dimensional space using analytical method(i.e., Hirtoa method) and numerical method. At the same time, in two distinct publications, Folkes et al. [12] studied the two-dimensional interaction of ion-acoustic solitons and Nishida et al. [13] investigated the oblique collision of plane ion-acoustic solitons, thus the two independent research groups further studied the interesting physical phenomenon of resonantly interacting solitary waves in the nonlinear model of ion-acoustic waves. Ohkuma and Wadati [14] systematically investigated how the phase shift coefficients influence the form of resonant solitary solutions of the KP equation, where the infinitely small and large phase shift coefficients result in soliton resonances. 
The above mentioned ideas and methods are applicable to a large number of nonlinear partial differential equations. In this work we study the so-called Mel'nikov equation, more precisely, the following system of coupled nonlinear partial differential equations:

$$
\begin{aligned}
& 3 u_{y y}-u_{x t}-\left[3 u^{2}+u_{x x}+\kappa|\psi|^{2}\right]_{x x}=0 \\
& i \psi_{y}=u \psi+\psi_{x x}
\end{aligned}
$$

where $\kappa$ is a constant, $\psi$ is a complex function of $x, y$, and $t$, which stands for the short-wave component, and $u$ is a real function of $x, y$, and $t$, which stands for the long-wave component.

The Mel'nikov equation (1) describes the interaction between long- and shortwavepackets in the two-dimensional space [15,16,17]. Additionally, there is another viewpoint of Mel'nikov [18], namely that the equation (1) is a coupled form of KP and NLS equations. In what follows we briefly discuss the results obtained in a series of works that have analyzed different types of localized solutions of the Mel'nikov equation. The general bright and dark solitary wave solutions of the Mel'nikov equation have been reported by Hase et al. [19] and Lakshmanan et al. [20] have investigated the exponentially localized solutions. The rational localized solutions have been calculated by $\mathrm{Mu}$ et al. [21], Deng et al. [22] have used the ansatz technique to derive lump and mixed rogue wave solutions, Sun et al. [23] have discussed the semi-rational solutions that describe the resonant collision between lumps and solitons, Zhang et al. [24] have reported the so-called hybrid solutions, and Rao et al. [25] have investigated two families of semi-rational solutions of Mel'nikov equation using the KP-hierarchy reduction method and the Hirota direct method combined with a perturbative expansion technique. Recently, twodimensional coherent structures of lump-soliton solutions of the Mel'nikov equation have been investigated by Liu et al. [26]. We also point out that families of exact solutions and the interaction scenarious between the corresponding waveforms have been recently investigated for other nonlinear partial differential equations that are relevant in several physics and engineering settings; see, for example, Refs. $[27,28,29,30,31,32]$.

In this paper, the Hirota direct method is used in order to obtain the exact solutions of the Mel'nikov equation (1) that describe the resonant collision scenarios between breathers and one dark line solitons and between lumps and one dark line solitons. We also investigate the resonant coilisions of breathers and lumps with two dark line solitons and the resonant collisions of breathers with higher-order dark line solitons, namely, the four dark line solitons. This work is organized as follows. In Section (2), we focus on deriving the exact solution describing the resonant collision between the breather and one dark line soliton and between the lump and one dark line soliton. We investigate in Section 3 the resonant collision of the breather with two dark line solitons, and of the lump with two dark line solitons. Then, in Section 4, we analyze the resonant collision among the breather and four dark line solitons. Finally, a summary and a brief discussion of the obtained results are given in Section 5 . 


\section{Resonant collision of a breather/lump and a dark line soliton}

In this Section, we study the resonant collision among a breather and a dark line soliton and the resonant collision of a lump and a dark line soliton based on the solutions (82) given in Appendix. We point out that the latter resonant collision is a limit case of the former one.

2.1 The resonant collision among a breather and a dark line soliton

Setting $N=3$ in Eq. (84) given in Appendix, the mixed solution consisting of a breather and a soliton can be derived as:

$$
\psi_{3}=\sqrt{2} \frac{G_{3}}{F_{3}}, \quad u_{3}=\left(2 \ln F_{3}\right)_{x x},
$$

where

$$
\begin{aligned}
& F_{3}=1+f_{31}+f_{32}+f_{33} \\
& G_{3}=1+g_{31}+g_{32}+g_{33}
\end{aligned}
$$

and

$$
\begin{aligned}
& f_{31}=e^{\eta_{1}}+e^{\eta_{2}}+e^{\eta_{3}}, \quad f_{32}=A_{12} e^{\eta_{1}+\eta_{2}}+A_{13} e^{\eta_{1}+\eta_{3}}+A_{23} e^{\eta_{2}+\eta_{3}}, \\
& f_{33}=A_{123} e^{\eta_{1}+\eta_{2}+\eta_{3}}, \quad g_{31}=c_{1} e^{\eta_{1}}+c_{2} e^{\eta_{2}}+c_{3} e^{\eta_{3}}, \\
& g_{32}=A_{12} c_{1} c_{2} e^{\eta_{1}+\eta_{2}}+A_{13} c_{1} c_{3} e^{\eta_{1}+\eta_{3}}+A_{23} c_{2} c_{3} e^{\eta_{2}+\eta_{3}}, \\
& g_{33}=A_{123} c_{123} e^{\eta_{1}+\eta_{2}+\eta_{3}}, \\
& \eta_{j}=k_{j} x+p_{j} y-\omega_{j} t+\eta_{j}^{0}, \\
& A_{j l}=\frac{k_{j} k_{l}\left(k_{j}-k_{l}\right)^{2}+\left(p_{l} k_{j}-p_{j} k_{l}\right)^{2}}{k_{j} k_{l}\left(k_{j}+k_{l}\right)^{2}+\left(p_{l} k_{j}-p_{j} k_{l}\right)^{2}}, \quad c_{j}=\frac{i p_{j}+k_{j}^{2}}{i p_{j}-k_{j}^{2}},
\end{aligned}
$$

and $A_{j l m}=A_{j l} A_{j m} A_{l m}, c_{j l m}=c_{j} c_{l} c_{m}$. Here the parameters $p_{j}, k_{j}, \omega_{j}$ meet the dispersion relation:

$$
\omega_{j}=\frac{\left(k_{j}^{4}-3 p_{j}^{2}\right)\left(k_{j}^{4}+p_{j}^{2}\right)-4 \kappa k_{j}^{4}}{\left(k_{j}^{4}+p_{j}^{2}\right) k_{j}}
$$

and $p_{1,2}, k_{1,2}, \omega_{1,2}, \eta_{1,2}^{0}$ further satisfy the parameter constraint in Eq. (87) given in Appendix while the parameters $p_{3}, k_{3}, \omega_{3}, \eta_{3}^{0}$ are real.

For a more general choices of the parameters $p_{j}, k_{j}, \omega_{j}(j=1,2,3)$, this solution demonstrates the elastic collision of a breather and a dark line soliton, which is shown in Fig. 1. According to the evolution graphs plotted in Fig. 1, we observe that the shapes, velocities, and amplitudes remain unchanged after collision. This conclusion is verified by the following asymptotic analysis.

To consider the key features of the collision between the breather and the dark line soliton, we focus on the asymptotic analysis of the dark line soliton and the breather. For this purpose, we rewrite the solution (2) into the following form:

$$
\psi_{3}=\frac{G_{3}}{F_{3}}, \quad u_{3}=\left(2 \ln F_{3}\right)_{x x}
$$




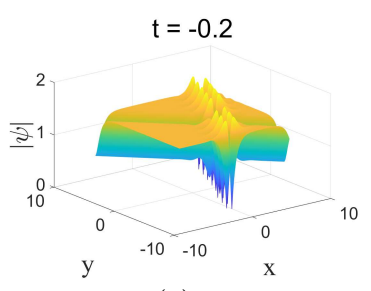

(a)

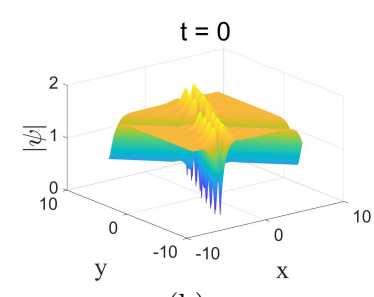

(b)

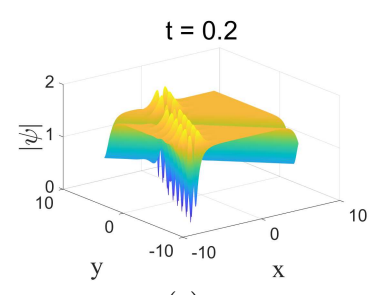

(c)

Fig. 1 The graphs showing the evolution of one dark line soliton and one breather with the following set of parameters: $\eta_{1}^{0}=0, \eta_{2}^{0}=0, \eta_{3}^{0}=0, \lambda_{11}=\frac{7}{4}, \lambda_{12}=\frac{1}{2}, a_{1}=-\frac{1}{2}, b_{1}=-1, q_{31}=$ $1, q_{32}=-\frac{1}{2}$.

and

$$
\eta_{1}=\eta_{1 R}+i \eta_{1 I}, \quad A_{13}=\rho_{13} e^{i \phi_{13}},
$$

where $\rho_{13}, \phi_{13}, \eta_{1 R}$, and $\eta_{1 I}$ are real. Under this condition, $F_{3}$ and $G_{3}$ could be simplified to the following triangular forms:

$$
\begin{aligned}
F_{3}= & \sqrt{A_{12}} \cosh \left(\eta_{1 R}+\ln \sqrt{A_{12}}\right)+\cos \eta_{1 I} \\
& +\rho_{13} e^{\eta_{3}}\left[\sqrt{A_{12}} \cosh \left(\eta_{1 R}+\ln \sqrt{A_{123}}\right)+\cos \left(\eta_{1 I}+\phi_{13}\right)\right] \\
G_{3}= & \sqrt{A_{12}} \cosh \left(\eta_{1 R}+\ln \sqrt{A_{12}}+i \phi_{c_{1}}\right)+\cosh \left(\eta_{1 I} i+\ln \rho_{c_{1}}\right)+\rho_{13} c_{3} e^{\eta_{3}} . \\
& {\left[\sqrt{A_{12}} \cosh \left(\eta_{1 R}+i \phi_{c_{1}}+\ln \sqrt{A_{123}}\right)+\cosh \left(\left(\eta_{1 I}+\phi_{13}\right) i+\ln \rho_{c_{1}}\right)\right], }
\end{aligned}
$$

where the subscripts $R$ and $I$ represent the real and imaginary parts of a given parameter or a function. From this expression, we can obtain that the dark line soliton denoted as $\mathbf{S}$ moves along $\eta_{3} \approx 0$ and the breather denoted as $\mathbf{B}$ propagates along $\eta_{1 R} \approx 0$. Then the asymptotic expressions for dark line soliton $\psi_{\mathbf{S}}$ and the breather soliton $\psi_{\mathbf{B}}$ are as follows.

Before collision:

The dark line soliton $\left(\eta_{3} \approx 0, \eta_{1 R} \rightarrow-\infty\right)$ :

$$
\overline{\mathrm{s}} \approx \sqrt{2 c_{3}} \frac{1+c_{3} e^{\eta_{3}}}{1+e^{\eta_{3}}}
$$

The breather $\left(\eta_{1 R} \approx 0, \eta_{3} \rightarrow+\infty\right)$ :

$\overline{\mathbf{B}} \approx \sqrt{2} c_{3} \frac{\sqrt{A_{12}} \cosh \left(\eta_{1 R}+i \phi_{c_{1}}+\ln \sqrt{A_{123}}\right)+\cosh \left[\left(\eta_{1 I}+\phi_{13}\right) i+\ln \rho_{c_{1}}\right]}{\sqrt{A_{12}} \cosh \left(\eta_{1 R}+\ln \sqrt{A_{123}}\right)+\cos \left(\eta_{1 I}+\phi_{13}\right)} ;$

After collision:

The dark line soliton $\left(\eta_{3} \approx 0, \eta_{1 R} \rightarrow+\infty\right)$ :

$$
\stackrel{+}{\mathbf{S}} \approx \sqrt{2 c_{3}} \frac{1+c_{3} e^{\eta_{3}+2 \ln \rho_{13}}}{1+e^{\eta_{3}+2 \ln \rho_{13}}}
$$


The breather $\left(\eta_{1 R} \approx 0, \eta_{3} \rightarrow-\infty\right)$ :

$$
\underset{\mathbf{B}}{+} \approx \sqrt{2} \frac{\sqrt{A_{12}} \cosh \left(\eta_{1 R}+i \phi_{c_{1}}+\ln \sqrt{A_{12}}\right)+\cosh \left(\eta_{1 I} i+\ln \rho_{c_{1}}\right)}{\sqrt{A_{12}} \cosh \left(\eta_{1 R}+\ln \sqrt{A_{12}}\right)+\cos \eta_{1 I}} .
$$

From these asymptotic expressions, we obtain that the phase shifts of soliton $\Delta \Phi_{\mathrm{S}}$ and breather $\Delta \Phi_{\mathrm{B}}$ are, respectively,

$$
\Delta \Phi_{\mathbf{S}}=2 \ln \rho_{13}, \quad \Delta \Phi_{\mathbf{B}}=\ln \rho_{13},
$$

where as we have defined in Eq. (7), $\rho_{13}$ represents the modulus of $A_{13}$. To transform the elastic collision into a resonant collision, one has to let $\Delta \Phi_{\mathbf{S}}, \Delta \Phi_{\mathbf{B}}$ be infinitely large, namely, $\ln \rho_{13} \rightarrow \infty$, which can be achieved by taking $A_{13}=0$ or $A_{13} \rightarrow \infty$. To simplify the resonant collision when $A_{13}=0$, we take the parameter transformations

$$
\begin{array}{ll}
k_{1}=\lambda_{11}+\lambda_{12} i, & p_{1}=2\left(\lambda_{11}+\lambda_{12} i\right)\left(a_{1}+b_{1} i\right) \\
k_{2}=\lambda_{11}-\lambda_{12} i, & p_{2}=2\left(\lambda_{11}-\lambda_{12} i\right)\left(a_{1}-b_{1} i\right) \\
k_{3}=2 q_{31}, & p_{3}=-4 q_{31} q_{32},
\end{array}
$$

where $\lambda_{11}, \lambda_{12}, q_{31}, q_{32}$ are free real parameters. Then the expression of $A_{13}$ is rewritten as

$$
A_{13}=\frac{N_{13} N_{23}}{D_{13} D_{23}},
$$

where

$$
\begin{aligned}
& N_{13}=\left(2 q_{32}+\lambda_{12}+2 a_{1}\right) i+\lambda_{11}-2 q_{31}-2 b_{1} \\
& N_{23}=\left(2 q_{32}-\lambda_{12}+2 a_{1}\right) i-\lambda_{11}+2 q_{31}-2 b_{1} \\
& D_{13}=\left(2 q_{32}+\lambda_{12}+2 a_{1}\right) i+\lambda_{11}+2 q_{31}-2 b_{1} \\
& D_{23}=\left(2 q_{32}-\lambda_{12}+2 a_{1}\right) i-\lambda_{11}-2 q_{31}-2 b_{1} .
\end{aligned}
$$

If $A_{13}=0$, the numerator should be zero, which infers to $N_{13}=0, N_{23}=0$, that is

$$
\begin{aligned}
& \left(2 q_{32}+\lambda_{12}+2 a_{1}\right) i+\lambda_{11}-2 q_{31}-2 b_{1}=0 \\
& \left(2 q_{32}-\lambda_{12}+2 a_{1}\right) i-\lambda_{11}+2 q_{31}-2 b_{1}=0,
\end{aligned}
$$

namely, the resonant condition $A_{13}=0$ is equivalent to

$$
\left\{\begin{array} { l } 
{ q _ { 3 1 } = \frac { 1 } { 2 } \lambda _ { 1 1 } - b _ { 1 } } \\
{ q _ { 3 2 } = - \frac { 1 } { 2 } \lambda _ { 1 2 } - a _ { 1 } }
\end{array} \text { or } \quad \left\{\begin{array}{l}
q_{31}=\frac{1}{2} \lambda_{11}+b_{1} \\
q_{32}=\frac{1}{2} \lambda_{12}-a_{1}
\end{array} .\right.\right.
$$

By submitting the resonant collision condition into the solution (6), one obtains the explicit expressions of the resonant collision solution as

$$
\psi_{3 r}=\sqrt{2} \frac{G_{3 r}}{F_{3 r}}, \quad u_{3 r}=\left(2 \ln F_{3 r}\right)_{x x},
$$

with

$$
\begin{aligned}
& F_{3 r}=1+2 e^{\eta_{1 R}} \cos \eta_{1 I}+e^{\eta_{3}}+A_{12} e^{2 \eta_{1 R}} \\
& G_{3 r}=1+2 e^{\eta_{1 R}+i \phi_{c_{1}}} \cosh \left(i \eta_{1 I}+\ln \rho_{c_{1}}\right)+c_{3} e^{\eta_{3}}+A_{12} e^{2 \eta_{1 R}+2 i \phi_{c_{1}}} .
\end{aligned}
$$

Upon the trajectories where the breather and the dark line soliton are moving along, the resonant collision described by Eq. (19) can be classified into two distinct 
types:

(1) Obliquely resonant collision of a dark line soliton and a breather when $\frac{k_{1 R}}{p_{1 R}} \neq \frac{k_{3}}{p_{3}}$. Thus, when the condition $\frac{k_{1 R}}{p_{1 R}} \neq \frac{k_{3}}{p_{3}}$ is satisfied, the breather will obliquely intersect with the dark line soliton in the $(x, y)$ plane.

Before collision:

The dark line soliton $\left(\eta_{3} \approx 0, \eta_{1 R} \rightarrow-\infty\right)$ :

$$
\overline{\mathrm{s}} \approx \sqrt{2} \frac{1+c_{3} e^{\eta_{3}}}{1+e^{\eta_{3}}}
$$

The breather $\left(\eta_{1 R} \approx 0, \eta_{3} \rightarrow+\infty\right)$ :

$$
\stackrel{+}{\mathbf{B}} \approx \sqrt{2} c_{3}
$$

After collision:

The dark line soliton $\left(\eta_{3} \approx 0, \eta_{1 R} \rightarrow+\infty\right)$ :

$$
\stackrel{+}{\mathrm{s}} \approx \sqrt{2}
$$

The breather $\left(\eta_{1 R} \approx 0, \eta_{3} \rightarrow-\infty\right)$ :

$$
\overline{\mathbf{B}} \approx \sqrt{2} \frac{\sqrt{A_{12}} \cosh \left(\eta_{1 R}+i \phi_{c_{1}}+\frac{1}{2} \ln A_{12}\right)+\cosh \left(\eta_{1 I} i+\ln \rho_{c_{1}}\right)}{\sqrt{A_{12}} \cosh \left(\eta_{1 R}+\frac{1}{2} \ln A_{12}\right)+\cos \eta_{1 I}} .
$$

The time evolution of the obliquely resonant collision of a dark line soliton and a breather is illustrated in Fig. 2 . It is seen that the breather only exists as $x \ll 0$ and disappears as $x \gg 0$ for given times, thus the breather is semi-localized in space and is not localized in time.

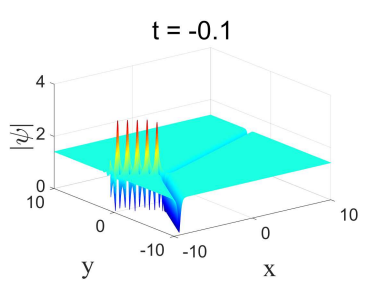

(a)

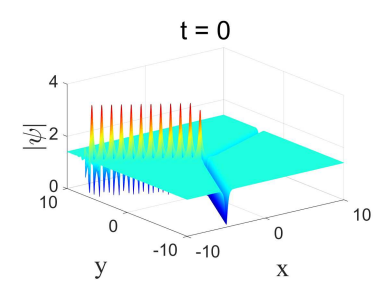

(b)

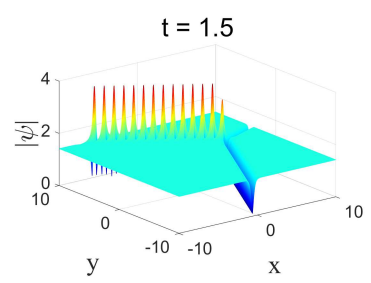

(c)

Fig. 2 The graph showing an oblique resonant collision among one dark line soliton and a breather with the following choice of the parameters: $\eta_{1}^{0}=0, \eta_{2}^{0}=0, \eta_{3}^{0}=0, \lambda_{11}=\frac{5}{3}, \lambda_{12}=$ $1, a_{1}=-\frac{3}{4}, b_{1}=-\frac{5}{2}$.

(2) Parallel resonant collision of a dark line soliton and a breather when $\frac{k_{1 R}}{p_{1 R}}=\frac{k_{3}}{p_{3}}$. Thus, when the condition $\frac{k_{1 R}}{p_{1 R}}=\frac{k_{3}}{p_{3}}$ holds, the breather and the dark line soliton will be parallel in the $(x, y)$ plane. Furthermore, from the identity $\frac{k_{1 R}}{p_{1 R}}=\frac{k_{3}}{p_{3}}$ it results the following equation:

$$
\frac{k_{1 R}}{p_{1 R}}-\frac{k_{3}}{p_{3}}=-\frac{\lambda_{12}\left(2 b_{1}+\lambda_{11}\right)}{2\left(\lambda_{11} a_{1}-\lambda_{12} b_{1}\right)\left(\lambda_{12}+2 a_{1}\right)}=0 .
$$


Since $2 b_{1}+\lambda_{11}=0$ gives rise to the denominator of expression of $A_{12}$ to be zero, thus Eq. (25) yields $\lambda_{12}=0$. Thus the solution (19) with parameters $q_{31}, q_{32}$ given by Eq. (18) and $\lambda_{12}=0$ illustrates a parallel resonant collision. Figure 3(a)(e) shows the time evolution of the parallel resonant collision among a breather and a dark line soliton. We see that the solution consists of a breather and a dark line soliton as $t \ll 0$, and it only comprises a dark line soliton as $t \gg 0$, namely, the breather fuses into the dark line soliton in this evolution process. Figure 3 (f) shows the intensity plot of the dark line soliton before collision (at time $t=-0.4$, blue dashed line) and after collision (at time $t=0.6$, red solid line). It is seen that the peak of the dark line soliton becomes deeper after collision, if we compare to the corresponding peak value before collision, and the breather waveform disappears into the constant background, which implies that after the resonant collision, both breather and dark line soliton completely vanish and a new dark line soliton is generated due to their interaction. Thus, we arrive at the conclusion that the breather in the parallel resonant collision is semi-localized in time and is not localized in the two-dimensional space.

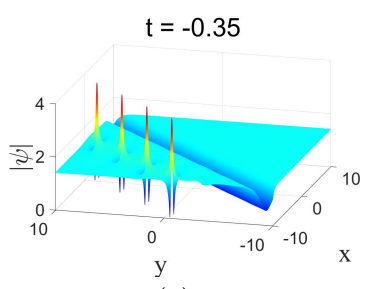

(a)

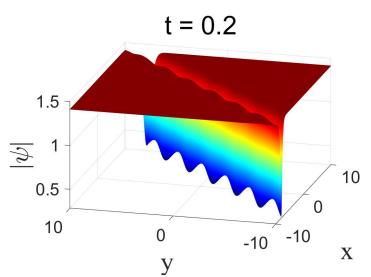

(d)

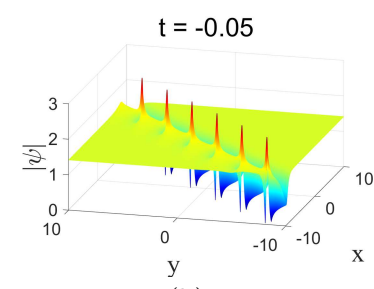

(b)

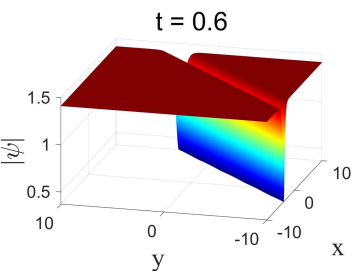

(e)

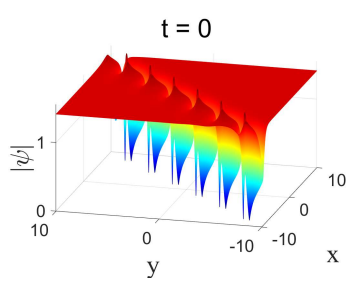

(c)

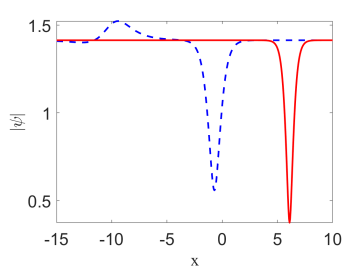

(f)

Fig. 3 The graph showing the solution describing the parallel resonant collision among a breather and a dark line soliton with the following choice of the parameters: $\eta_{1}^{0}=0, \eta_{2}^{0}=$ $0, \eta_{3}^{0}=0, \lambda_{11}=\frac{2}{3}, \lambda_{12}=0, a_{1}=-\frac{1}{2}, b_{1}=-\frac{3}{2}$. In particular, Fig. 3(f) is a sectional view of this evolution at $y=0$, where the blue dashed line shows the waveform before collision, at $t=-0.4$, while the red solid line shows the waveform after collision, at $t=0.6$.

2.2 The resonant collision among a lump and a dark line soliton

We now consider the resonant collision of a lump and a dark line soliton. To this end, we first obtain the solution describing the elastic collision of a lump and a dark line soliton. We substitute $k_{j}=k_{j} \epsilon, p_{j}=p_{j} \epsilon$ into Eq. (3), we take the limit 
$\epsilon \rightarrow 0$ of it, and then we simplify it with the variable transformation in Eq. (14). Thus the mixed solution consisting of a lump and a dark line soliton is given by:

$$
\psi_{3 l}=\sqrt{2} \frac{g_{3}}{f_{3}}, \quad u_{3 l}=\left(2 \ln f_{3}\right)_{x x}
$$

with

$$
\begin{aligned}
& f_{3}=\left(\left|\theta_{1}+a_{13}\right|^{2}+a_{12}\right) e^{\eta_{3}}+\left|\theta_{1}\right|^{2}+a_{12}, \\
& g_{3}=\left[\left(\theta_{1}+a_{13}+r_{1}\right)\left(\theta_{1}^{*}+a_{13}^{*}-r_{1}^{*}\right)+a_{12}\right] c_{3} e^{\eta_{3}}+\left(\theta_{1}+r_{1}\right)\left(\theta_{1}^{*}-r_{1}^{*}\right)+a_{12},
\end{aligned}
$$

where

$$
\begin{aligned}
a_{12} & =\frac{\lambda_{11}^{2}+\lambda_{12}^{2}}{4 b_{1}^{2}}, \quad a_{13}=\frac{N_{31}}{D_{31} D_{32}}, \quad r_{1}=\frac{\lambda_{12}-\lambda_{11} i}{a_{1}+b_{1} i}, \\
\theta_{1} & =k_{1} x+p_{1} y-\omega_{1 L} t, \quad \eta_{3}=k_{3} x+p_{3} y-\omega_{3} t+\eta_{3}^{0}
\end{aligned}
$$

with

$$
\begin{aligned}
& D_{31}=\left(q_{32}+a_{1}\right) i-q_{31}-b_{1}, \quad N_{31}=2 q_{31}\left(\lambda_{11}+\lambda_{12} i\right) \\
& D_{32}=\left(q_{32}+a_{1}\right) i+q_{31}-b_{1}, \\
& \omega_{1 L}=\frac{\left(48 a_{1}^{3} b_{1} i-48 a_{1} b_{1}^{3} i+12 a_{1}^{4}-72 a_{1}^{2} b_{1}^{2}+12 b_{1}^{4}+\kappa\right)\left(\lambda_{11}+\lambda_{12} i\right)}{\left(a_{1}+b_{1} i\right)^{2}} .
\end{aligned}
$$

In this solution, the lump moves along $\left|\theta_{1}\right| \approx 0$. As $\eta_{3} \rightarrow-\infty$, the solution $\psi_{3 l}$ in (26) becomes:

$$
\psi_{3 l}^{-}=\sqrt{2} \frac{\left(\theta_{1}+r_{1}\right)\left(\theta_{1}^{*}-r_{1}^{*}\right)+a_{12}}{\left|\theta_{1}\right|^{2}+a_{12}},
$$

and the lump is centered at

$$
\left|\theta_{1}\right|=0
$$

However, as $\eta_{3} \rightarrow+\infty$, the solution $\psi_{3 l}$ in (26) becomes:

$$
\psi_{3 l}^{+}=\sqrt{2} \frac{\left[\left(\theta_{1}+a_{13}+r_{1}\right)\left(\theta_{1}^{*}+a_{13}^{*}-r_{1}^{*}\right)+a_{12}\right] c_{3}}{\left|\theta_{1}+a_{13}\right|^{2}+a_{12}},
$$

and the lump is centered at

$$
\left|\theta_{1}+a_{13}\right|=0
$$

From the above analysis, we obtain that the phase shift of the lump after collision is $\Delta \Phi_{3 L}=a_{13}$. Then the elastic collision of a lump and a dark line soliton, which is described by the solution (26), can be transformed into a resonant collision when $\Delta \Phi_{3 L}=a_{13} \rightarrow \infty$. Thus we get the following relation that is termed as the resonant condition:

$$
\begin{aligned}
& \left(q_{32}+a_{1}\right) i-q_{31}-b_{1}=0, \\
& \left(q_{32}+a_{1}\right) i+q_{31}-b_{1}=0 .
\end{aligned}
$$

By solving this equation, one obtains that:

$$
\left\{\begin{array} { l } 
{ q _ { 3 1 } = - b _ { 1 } } \\
{ q _ { 3 2 } = - a _ { 1 } }
\end{array} \quad \text { or } \quad \left\{\begin{array}{l}
q_{31}=b_{1} \\
q_{32}=-a_{1}
\end{array}\right.\right.
$$


Eventually, we get the solution consisting of a lump and a dark line soliton, whose form is

$$
\psi_{3 l}=\sqrt{2} \frac{g_{3 l}}{f_{3 l}}, \quad u_{3 l}=\left(2 \ln f_{3 l}\right)_{x x}
$$

with

$$
f_{3 l}=e^{\eta_{3}}+\left|\theta_{1}\right|^{2}+a_{12}, \quad g_{3 l}=c_{3} e^{\eta_{3}}+\left(\theta_{1}+r_{1}\right)\left(\theta_{1}^{*}-r_{1}^{*}\right)+a_{12},
$$

where the meanings of the various notations are exhibited in Eqs. (28)-(29).

Figure 4 shows several particular graphs of resonant collision of one dark line soliton and a lump. We see that when the resonant collision occurs, the lump dissolves into the dark line soliton.

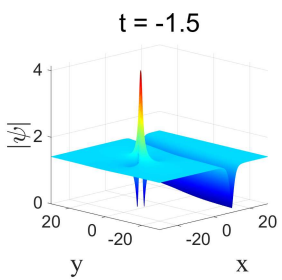

(a)

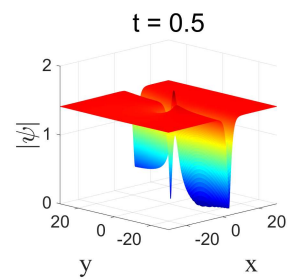

(b)

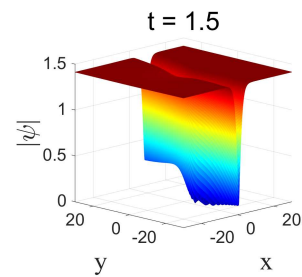

(c)

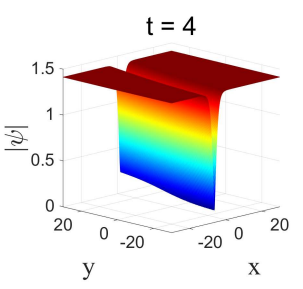

(d)

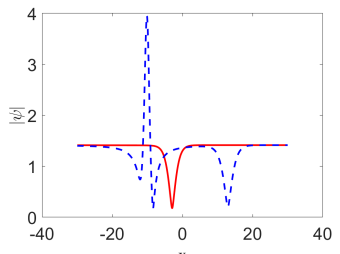

(e)

Fig. 4 The graphs exhibiting the resonant collision among a lump and one dark line soliton with the following set of parameters: $\eta_{3}^{0}=0, \lambda_{11}=\frac{1}{6}, \lambda_{12}=3, a_{1}=-\frac{1}{15}, b_{1}=-\frac{1}{2}$. Particularly, Fig. 4(e) shows the shapes of the waveforms before collision and after collision: the blue dashed line shows the shape at $t=-1.5$, before collision, and the red solid line shows the shape at $t=4$, after collision.

\section{Resonant collision of a breather/lump and two dark line solitons}

In this Section, we first consider the obliquely and parallel resonant collision of a breather and two dark line solitons. We arrive at the conclusion that when the breather and the two dark line solitons obliquely intersect each other we get that the breather is completely localized in space and is not localized in time, and when the breather and the two dark line solitons are parallel to each other, we get that the breather is completely localized in time and is not localized in space. Then we consider the resonant collision of a lump and two dark line solitons as the limit case of the resonant collision of a breather and two dark line solitons. 
3.1 Resonant collision of a breather and two dark line solitons

In this case, setting $N=4$ in Eq. (84), we could express the mixed solution consisting of a breather and two dark line solitons as:

$$
\psi_{4}=\sqrt{2} \frac{G_{4}}{F_{4}}, \quad u_{4}=\left(2 \ln F_{4}\right)_{x x}
$$

where

$$
\begin{aligned}
& F_{4}=f_{41}+A_{12} e^{2 \eta_{1 R}} f_{42}+2 e^{\eta_{1 R}} f_{43} \\
& G_{4}=g_{41}+A_{12} e^{2 \eta_{1 R}+2 i \phi_{c_{1}}} g_{42}+2 e^{\eta_{1 R}+i \phi_{c_{1}}} g_{43}
\end{aligned}
$$

with

$$
\begin{aligned}
f_{41}= & 1+e^{\eta_{3}}+e^{\eta_{4}}+A_{34} e^{\eta_{3}+\eta_{4}}, \\
f_{42}= & 1+\rho_{13}^{2} e^{\eta_{3}}+\rho_{14}^{2} e^{\eta_{4}}+A_{34} \rho_{13}^{2} \rho_{14}^{2} e^{\eta_{3}+\eta_{4}}, \\
f_{43}= & \cos \eta_{1 I}+\rho_{13} e^{\eta_{3}} \cos \left(\eta_{1 I}+\phi_{13}\right)+\rho_{14} e^{\eta_{4}} \cos \left(\eta_{1 I}+\phi_{14}\right) \\
& +A_{34} \rho_{13} \rho_{14} e^{\eta_{3}+\eta_{4}} \cos \left(\eta_{1 I}+\phi_{13}+\phi_{14}\right), \\
g_{41}= & 1+c_{3} e^{\eta_{3}}+c_{4} e^{\eta_{4}}+A_{34} c_{3} c_{4} e^{\eta_{3}+\eta_{4}}, \\
g_{42}= & 1+\rho_{13}^{2} c_{3} e^{\eta_{3}}+\rho_{14}^{2} c_{4} e^{\eta_{4}}+A_{34} \rho_{13}^{2} \rho_{14}^{2} c_{3} c_{4} e^{\eta_{3}+\eta_{4}}, \\
g_{43}= & \cosh \left(\eta_{1 I} i+\ln \rho_{c_{1}}\right)+\rho_{13} c_{3} e^{\eta_{3}} \cosh \left[\left(\eta_{1 I}+\phi_{13}\right) i+\ln \rho_{c_{1}}\right] \\
& +\rho_{14} c_{4} e^{\eta_{4}} \cosh \left[\left(\eta_{1 I}+\phi_{14}\right) i+\ln \rho_{c_{1}}\right] \\
& +A_{34} \rho_{13} \rho_{14} c_{3} c_{4} e^{\eta_{3}+\eta_{4}} \cosh \left[\left(\eta_{1 I}+\phi_{13}+\phi_{14}\right) i+\ln \rho_{c_{1}}\right],
\end{aligned}
$$

and

$$
\begin{aligned}
A_{l m} & =\frac{k_{m} k_{l}\left(k_{m}-k_{l}\right)^{2}+\left(p_{l} k_{m}-p_{m} k_{l}\right)^{2}}{k_{m} k_{l}\left(k_{m}+k_{l}\right)^{2}+\left(p_{l} k_{m}-p_{m} k_{l}\right)^{2}}, \quad c_{j}=\frac{i p_{j}+k_{j}^{2}}{i p_{j}-k_{j}^{2}}, \\
\eta_{j} & =k_{j} x+p_{j} y-\omega_{j} t+\eta_{j}^{0}, \quad \eta_{1 R}=\operatorname{Re} \eta_{1}, \quad \eta_{1 I}=\operatorname{Im} \eta_{1}, \\
\rho_{1 j} & =\left|A_{1 j}\right|, \quad \phi_{1 j}=\arg A_{1 j}, \quad \rho_{c_{1}}=\left|c_{1}\right|, \quad \phi_{c_{1}}=\arg c_{1},
\end{aligned}
$$

and $p_{j}, k_{j}, \omega_{j}$ meeting the dispersion relation shown in Eq. (5).

Moreover, in order to facilitate the analysis, we group the variable transformations extended from (14) as follows

$$
\begin{array}{ll}
k_{1}=\lambda_{11}+\lambda_{12} i, & p_{1}=2\left(\lambda_{11}+\lambda_{12} i\right)\left(a_{1}+b_{1} i\right), \\
k_{2}=\lambda_{11}-\lambda_{12} i, & p_{2}=2\left(\lambda_{11}-\lambda_{12} i\right)\left(a_{1}-b_{1} i\right), \\
k_{3}=2 q_{31}, & p_{3}=-4 q_{31} q_{32}, \\
k_{4}=2 q_{41}, & p_{4}=-4 q_{41} q_{42} .
\end{array}
$$

We substitute these transformation into (36), and we finish the construction of the solution describing the elastic collision among a breather and two dark line solitons including the free real parameters $\lambda_{11}, \lambda_{12}, a_{1}, b_{1}, q_{31}, q_{32}, q_{41}, q_{42}$, and $\eta_{j}^{0}, j=1,2,3,4$. Figure 5 shows a typical ellastic collision of a breather and two dark line solitons.

In Section 2.1, we have analyzed the collision behavior among a breather and one soliton waveform using the asymptotic analysis and we have obtained the phase shift of the breather denoted as $\Delta \Phi_{\mathbf{B}}$ and of the soliton denoted as $\Delta \Phi_{\mathbf{S}}$. In the same way, it is not so difficult to get that the solution expressing the collision 


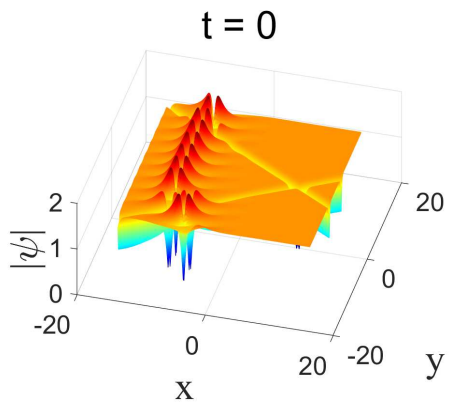

(a)

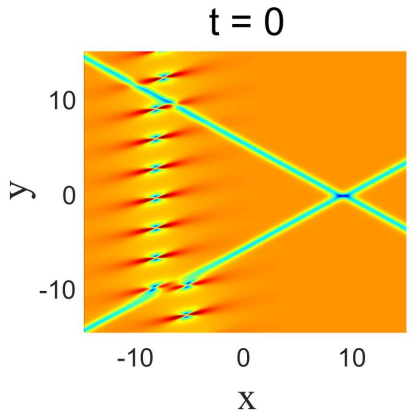

(b)

Fig. 5 The graphs demonstrating the elastic collision among a breather and two dark line solitons with the following choice of the parameters: $\eta_{1}^{0}=4, \eta_{2}^{0}=4, \eta_{3}^{0}=-25, \eta_{4}^{0}=-25, \lambda_{11}=$ $\frac{1}{2}, \lambda_{12}=\frac{1}{2}, a_{1}=-1, b_{1}=-1, q_{31}=\frac{3}{2}, q_{32}=-\frac{4}{5}, q_{41}=\frac{3}{2}, q_{42}=\frac{4}{5}$.

behavior among a breather and two dark line solitons has almost the same form, where the corresponding phase shifts are

$$
\Delta \Phi_{\mathbf{S}}^{3}=2 \ln \rho_{13}, \quad \Delta \Phi_{\mathbf{S}}^{4}=2 \ln \rho_{14}, \quad \Delta \Phi_{\mathbf{B}}^{1}=\ln \rho_{13} \rho_{14}
$$

where $\Delta \Phi_{\mathbf{S}}^{3}, \Delta \Phi_{\mathbf{S}}^{4}$, and $\Delta \Phi_{\mathbf{B}}^{1}$ are the phase shifts of the soliton along $\eta_{3} \approx 0$, $\eta_{4} \approx 0$, and of the breather along $\eta_{1 R} \approx 0$, respectively.

To transform the elastic collision in Eq. (36) into a resonant collision, we should let $\Delta \Phi_{\mathbf{S}}^{3}, \Delta \Phi_{\mathbf{S}}^{4}$, and $\Delta \Phi_{\mathbf{B}}^{1}$ be infinitely large, in other words, $\ln \rho_{13} \rightarrow \infty, \ln \rho_{14} \rightarrow$ $\infty$, which demands for taking $A_{13}=0$ and $A_{14}=0$. Actually, the parameter constraint satisfying $A_{13}=0$ and $A_{14}=0$ is almost the same as what we have obtained in Eq. (18), that is

$$
\left\{\begin{array} { l } 
{ q _ { 3 1 } = \frac { 1 } { 2 } \lambda _ { 1 1 } - b _ { 1 } } \\
{ q _ { 3 2 } = - \frac { 1 } { 2 } \lambda _ { 1 2 } - a _ { 1 } }
\end{array} \text { and } \quad \left\{\begin{array}{l}
q_{41}=\frac{1}{2} \lambda_{11}+b_{1} \\
q_{42}=\frac{1}{2} \lambda_{12}-a_{1} .
\end{array}\right.\right.
$$

Under this constraint, we simplify the solution of elastic collision (36) to obtain the solution describing the resonant collision among a breather and two dark line solitons:

$$
\phi_{4 r}=\sqrt{2} \frac{G_{4 r}}{F_{4 r}}, \quad u_{4 r}=\left(2 \ln F_{4 r}\right)_{x x},
$$

where

$$
\begin{aligned}
F_{4 r}= & 1+2 e^{\eta_{1 R}} \cos \eta_{1 I}+e^{\eta_{3}}+e^{\eta_{4}}+A_{12} e^{2 \eta_{1 R}}+A_{34} e^{\eta_{3}+\eta_{4}}, \\
G_{4 r}= & 1+2 e^{\eta_{1 R}+i \phi_{c_{1}}} \cosh \left(\eta_{1 I} i+\ln \rho_{c_{1}}\right)+c_{3} e^{\eta_{3}}+c_{4} e^{\eta_{4}} \\
& +A_{12} e^{2 \eta_{1 R}+2 i \phi_{c_{1}}}+A_{34} c_{3} c_{4} e^{\eta_{3}+\eta_{4}} .
\end{aligned}
$$

In accordance with the trajectories of the breather and of the dark line solitons on which they are moving along, the resonant collisions described by the solution given in Eq. (43) are classified into two distinct types:

(1) Obliquely resonant collision of two dark line solitons and a breather when $\frac{k_{1 R}}{p_{1 R}} \neq \frac{k_{j}}{p_{j}}, j=3,4$. Thus when the conditions $\frac{k_{1 R}}{p_{1 R}} \neq \frac{k_{j}}{p_{j}}, j=3,4$ are 
satisfied, the breather will obliquely intersect with the dark line solitons in the $(x, y)$ plane.

Before collision:

The dark line soliton $\left(\eta_{3} \approx 0, \eta_{4} \rightarrow-\infty, \eta_{1 R} \rightarrow-\infty\right)$ :

$$
\psi_{\mathbf{S}}^{3-} \approx \sqrt{2} \frac{1+c_{3} e^{\eta_{3}}}{1+e^{\eta_{3}}}
$$

The dark line soliton $\left(\eta_{4} \approx 0, \eta_{3} \rightarrow-\infty, \eta_{1 R} \rightarrow-\infty\right)$ :

$$
\psi_{\mathbf{S}}^{4-} \approx \sqrt{2} \frac{1+c_{4} e^{\eta_{4}}}{1+e^{\eta_{4}}}
$$

The breather $\left(\eta_{1 R} \approx 0, \eta_{3} \rightarrow+\infty, \eta_{4} \rightarrow+\infty\right)$ :

$$
\psi_{\mathbf{B}}^{+} \approx \sqrt{2} c_{4} .
$$

After collision:

The dark line soliton $\left(\eta_{3} \approx 0, \eta_{4} \rightarrow+\infty, \eta_{1 R} \rightarrow+\infty\right)$ :

$$
\psi_{\mathbf{S}}^{3+} \approx \sqrt{2} c_{4} \frac{1+A_{34} c_{3} e^{\eta_{3}}}{1+A_{34} e^{\eta_{3}}}
$$

The dark line soliton $\left(\eta_{4} \approx 0, \eta_{3} \rightarrow+\infty, \eta_{1 R} \rightarrow+\infty\right)$ :

$$
\psi_{\mathbf{S}}^{4+} \approx \sqrt{2} c_{3} \frac{1+A_{34} c_{4} e^{\eta_{4}}}{1+A_{34} e^{\eta_{4}}}
$$

The breather $\left(\eta_{1 R} \approx 0, \eta_{3} \rightarrow-\infty, \eta_{4} \rightarrow-\infty\right)$ :

$$
\psi_{\mathbf{B}}^{-} \approx \sqrt{2} c_{3} .
$$

If we compare equations (45), (46), and (47) with equations (48), (49), and (50), we can summarize that after the resonant collision, the two dark line solitons, except for a phase shift $\Delta \Phi=\ln A_{34}$ between them, have no change in amplitude, velocity, and shape, which is accordance with what Fig. 6 indicates. We can see from this figure that the breather is remarkably localized in space and is not localized in time due to the the resonance.

(2) Parallel resonant collision of two dark line solitons and a breather when $\frac{k_{1 R}}{p_{1 R}}=\frac{k_{j}}{p_{j}}, j=3,4$. Thus, when the conditions $\frac{k_{1 R}}{p_{1 R}}=\frac{k_{j}}{p_{j}}, j=3,4$ are fulfilled, the breather will parallelly intersect the two dark line solitons in the $(x, y)$ plane. Furthermore, the constraint $\frac{k_{1 R}}{p_{1 R}}=\frac{k_{j}}{p_{j}}, j=3,4$ leads to the same result as that resulting from Eq. (25), namely, we obtain $\lambda_{12}=0$. Thus the solution (43) with the parameters $q_{31}, q_{32}, q_{41}, q_{42}$ given by Eq. (42) and $\lambda_{12}=0$ illustrates the parallel resonant collision. Figure 7 exhibits the dynamics of evolution of the parallel resonant collision among a breather and two dark line solitons. We see that both at $t \ll 0$ and at $t \gg 0$, the solution consists of two dark line solitons, which means that the resonant collision does not change the shape and the type of dark line solitons. However, within the colliding area, the solution displays a breather waveform, namely, the two dark line solitons join together to generate the breather and then the solution returns after a short period of time to that 


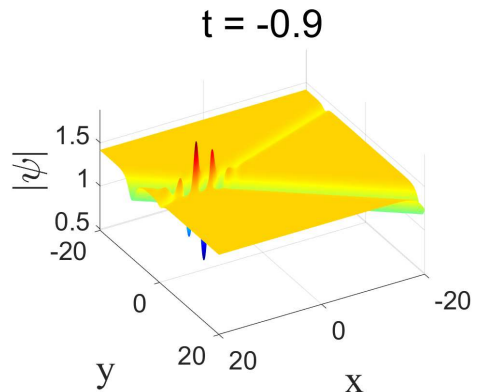

(a)

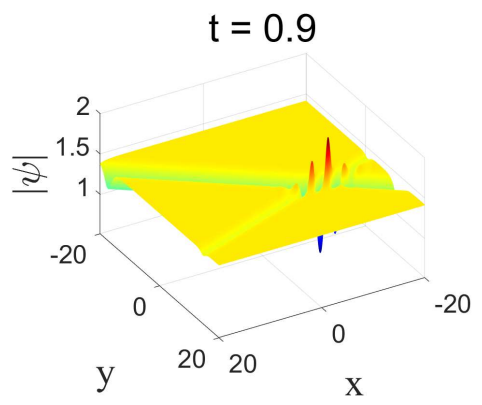

(c)

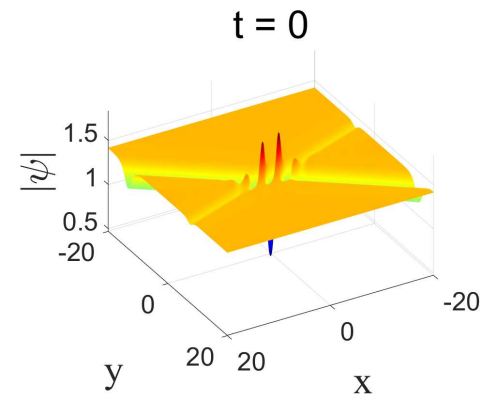

(b)

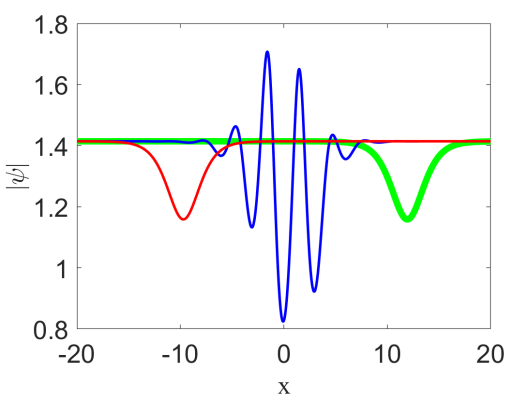

(d)

Fig. 6 The graphs of the solution demonstrating the oblique resonant collision among a breather and two dark line solitons with the following set of the parameters: $\eta_{1}^{0}=0, \eta_{2}^{0}=$ $0, \eta_{3}^{0}=0, \eta_{4}^{0}=0, \lambda_{11}=-\frac{1}{10}, \lambda_{12}=2, a_{1}=-\frac{1}{3}, b_{1}=\frac{5}{12}$. Particularly, Fig. 6(d) shows the shapes of solution describing the resonant collision at $y=0$, where the green, blue, and red lines show the shapes at $t=-2,0,2$, respectively.

corresponding to two dark line solitons. In other words, the breather in this figure is completely localized in time and is not localized in space. Figure $7(\mathrm{f})$ shows the sectional shape of the solution before collision (at time $t=-4$, the blue dashed line), during collision (at time $t=0$, the red solid line), and after collision (at time $t=4$, the green dashed line). We observe that before and after the resonant collision, the waveform of the two dark line soliton has no change in depth and shape, while during collision, the solution shows periodic oscillations, which means that the amplitude and shape of the two dark line soliton have no change, however, when collision occurs, they act together to produce a breather.

\subsection{Resonant collision of a lump and two dark line solitons}

We consider the resonant collision of a lump and two dark line solitons. To this aim, we first obtain the solution describing the elastic collision of a lump and two dark line solitons. We use the same method as in Section 2.2 and we get

$$
\psi_{4 l}=\sqrt{2} \frac{g_{4}}{f_{4}}, \quad u_{4 l}=\left(2 \ln f_{4}\right)_{x x},
$$




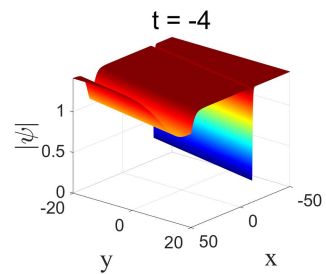

(a)

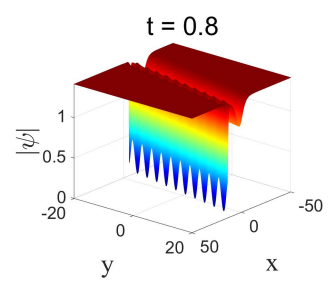

(d)

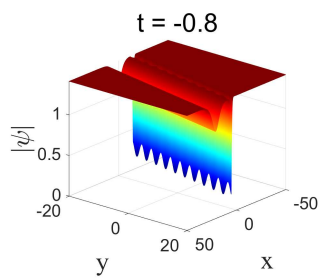

(b)

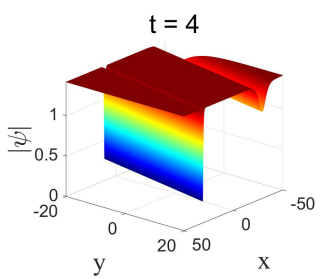

(e)

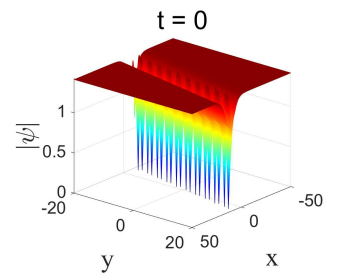

(c)

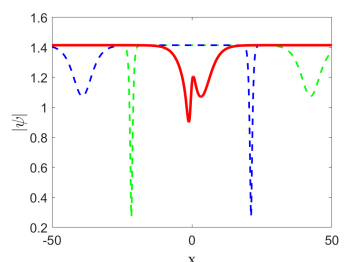

(f)

Fig. 7 The graphs of evolution of parallel resonant collision among one breather and two dark line solitons with the set of parameters: $\eta_{1}^{0}=0, \eta_{2}^{0}=0, \eta_{3}^{0}=0, \eta_{4}^{0}=0, \lambda_{11}=\frac{15}{14}, \lambda_{12}=$ $0, a_{1}=-\frac{1}{4}, b_{1}=-\frac{3}{4}$. Particularly, Fig. $7(\mathrm{f})$ shows the sectional shapes for three values of time, namely, at $t=-4$ (the blue dashed line), $t=0$ (the red solid line), and $t=4$ (the green dashed line).

and the constituents $f_{4}$ and $g_{4}$ are defined as

$$
\begin{aligned}
f_{4}= & A_{34}\left[a_{12}+\left|\theta_{1}+a_{13}+a_{14}\right|^{2}\right] e^{\theta_{3}+\theta_{4}}+\left(a_{12}+\left|\theta_{1}+a_{13}\right|^{2}\right) e^{\theta_{3}} \\
& +\left(a_{12}+\left|\theta_{1}+a_{14}\right|^{2}\right) e^{\theta_{4}}+\left|\theta_{1}\right|^{2}+a_{12}, \\
g_{4}= & A_{34}\left[a_{12}+\left(\theta_{1}+a_{13}+a_{14}+r_{1}\right)\left(\theta_{1}^{*}+a_{13}^{*}+a_{14}^{*}-r_{1}^{*}\right)\right] e^{\theta_{3}+\theta_{4}} \\
& +\left[a_{12}+\left(\theta_{1}+a_{13}+r_{1}\right)\left(\theta_{1}^{*}+a_{13}^{*}-r_{1}^{*}\right)\right] c_{3} e^{\theta_{3}} \\
& +\left[a_{12}+\left(\theta_{1}+a_{14}+r_{1}\right)\left(\theta_{1}^{*}+a_{14}^{*}-r_{1}^{*}\right)\right] c_{4} e^{\theta_{4}}+\left(\theta_{1}+r_{1}\right)\left(\theta_{1}^{*}-r_{1}^{*}\right)+a_{12},
\end{aligned}
$$

where

$$
\begin{array}{rlrl}
a_{12} & =\frac{\lambda_{11}^{2}+\lambda_{12}^{2}}{4 b_{1}^{2}}, \quad r_{1}=\frac{\lambda_{12}-\lambda_{11} i}{a_{1}+b_{1} i}, \quad a_{1 j}=\frac{N_{j 1}}{D_{j 1} D_{j 2}}, \\
c_{3} & =\frac{a_{1}+b_{1} i}{a_{1}-b_{1} i}, \quad c_{4}=\frac{a_{1}-b_{1} i}{a_{1}+b_{1} i}, & A_{34}=\frac{\left(q_{31}-q_{41}\right)^{2}+\left(q_{32}-q_{42}\right)^{2}}{\left(q_{31}+q_{41}\right)^{2}+\left(q_{32}-q_{42}\right)^{2}}, \\
\theta_{1} & =k_{1} x+p_{1} y-\omega_{1 L} t, \quad \theta_{l}=k_{l} x+p_{l} y-\omega_{l} t+\eta_{l}^{0}, l=3,4
\end{array}
$$

with

$$
\begin{aligned}
D_{j 1} & =\left(q_{j 2}+a_{1}\right) i-q_{j 1}-b_{1}, \quad N_{j 1}=2 q_{j 1}\left(\lambda_{11}+\lambda_{12} i\right) \\
D_{j 2} & =\left(q_{j 2}+a_{1}\right) i+q_{j 1}-b_{1}, \\
\omega_{1 L} & =\frac{\left(48 a_{1}{ }^{3} b_{1} i-48 a_{1} b_{1}{ }^{3} i+12 a_{1}{ }^{4}-72 a_{1}{ }^{2} b_{1}{ }^{2}+12 b_{1}{ }^{4}+\kappa\right)\left(\lambda_{11}+\lambda_{12} i\right)}{\left(a_{1}+b_{1} i\right)^{2}} .
\end{aligned}
$$


In this solution, the lump moves along $\left|\theta_{1}\right| \approx 0$. As $\theta_{3}, \theta_{4} \rightarrow-\infty$, the asymptotic form of the solution $\psi_{4 l}$ in Eq. (51) is given by

$$
\psi_{4 l}^{-} \approx \sqrt{2} \frac{\left|\theta_{1}+r_{1}\right|^{2}+a_{12}}{\left|\theta_{1}\right|^{2}+a_{12}},
$$

and the lump is centered at

$$
\left|\theta_{1}\right|=0 .
$$

Nevertheless, when $\theta_{3}, \theta_{4} \rightarrow+\infty$, the solution $\psi_{4 l}$ in Eq. (51) becomes

$$
\psi_{4 l}^{+} \approx \sqrt{2} \frac{\left|\theta_{1}+a_{13}+a_{14}+r_{1}\right|^{2}+a_{12}}{\left|\theta_{1}+a_{13}+a_{14}\right|^{2}+a_{12}},
$$

and the lump is centered at

$$
\left|\theta_{1}+a_{13}+a_{14}\right|=0
$$

From the above analysis, we obtain that the phase shift of the lump after collision is given by $\Delta \Phi_{4 L}=a_{13}+a_{14}$. Then the elastic collision of a lump and two dark line solitons in solution (51) will be transformed into a resonant collision when $\Delta \Phi_{4 L}=a_{13}+a_{14} \rightarrow \infty$, which leads to the following constraint conditions:

$$
\left\{\begin{array}{l}
q_{31}=-b_{1} \\
q_{32}=-a_{1}
\end{array}, \quad\left\{\begin{array}{l}
q_{41}=b_{1} \\
q_{42}=-a_{1}
\end{array} .\right.\right.
$$

Finally, we obtain the resonant solution consisting of a lump and a dark line soliton under above conditions, whose form is

$$
\psi_{4 l r}=\sqrt{2} \frac{g_{4 l r}}{f_{4 l r}}, \quad u_{4 l r}=\left(2 \ln f_{4 l r}\right)_{x x}
$$

with

$$
\begin{aligned}
& f_{4 l r}=a_{34} e^{\theta_{3}+\theta_{4}}+e^{\theta_{3}}+e^{\theta_{4}}+\left|\theta_{1}\right|^{2}+a_{12} \\
& g_{4 l r}=a_{34} c_{3} c_{4} e^{\theta_{3}+\theta_{4}}+c_{3} e^{\theta_{3}}+c_{4} e^{\theta_{4}}+\left(\theta_{1}+r_{1}\right)\left(\theta_{1}^{*}-r_{1}^{*}\right)+a_{12},
\end{aligned}
$$

where we have to note that all of the parameters in (59) are the same as those shown in (53) except for a change that $A_{34}$ becomes $a_{34}$ when taking the corresponding limit:

$$
a_{34}=\frac{4 b_{1}^{2}}{\lambda_{11}^{2}+\lambda_{12}^{2}} .
$$

Thus the solution describing the resonant collision among one lump and two dark line solitons is involving the parameters $\lambda_{11}, \lambda_{12}, a_{1}, b_{1}$, and $\eta_{j}^{0}, j=3,4$. Figure 8 exhibits the dynamics of evolution of this kind of collision. We see the interesting phenomenon that a lump splits out of one soliton and then dissolves into the other soliton during the collision process. Figure $8(\mathrm{f})$ exhibits the intensity plot of the solution before collision (at time $t=-6$, the green solid line), during collision (at time $t=0$, the red solid line), and after collision (at time $t=6$, the blue solid line), which reveals the fact that the soliton who emits the lump is decreasing its amplitude while the one who absorbs the lump is increasing its amplitude. 


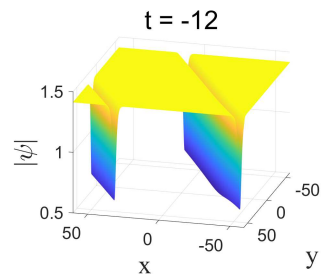

(a)

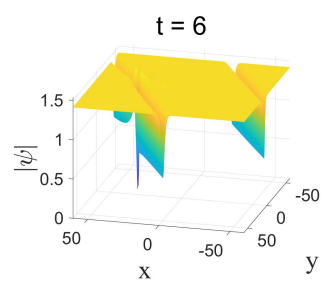

(d)

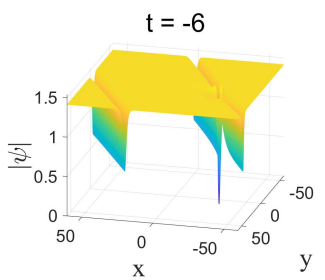

(b)

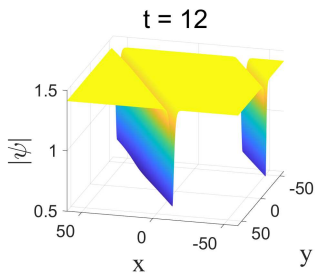

(e)

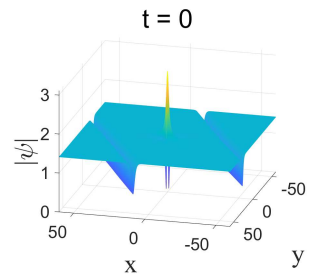

(c)

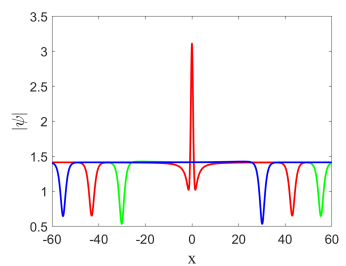

(f)

Fig. 8 The graphs of evolution of resonant collision among one lump and two dark line solitons with the following set of parameters: $\eta_{3}^{0}=-14 \pi, \eta_{4}^{0}=-14 \pi, \lambda_{11}=1, \lambda_{12}=\frac{1}{3}, a_{1}=\frac{3}{10}, b_{1}=$ $-\frac{3}{5}$. Particularly, Fig. 8(f) illustrates the sectional shapes of the corresponding waveforms at $t \stackrel{5}{=}-6$ (green line), $t=0$ (red line), and $t=6$ (blue line).

\section{Resonant collision of a breather and four dark line solitons}

In this Section, we study the resonant collision among a breather and four dark line solitons based on the solutions (82).

To this end, we first set $N=6$ in (84) to obtain the solution describing the elastic collision of a lump and four dark line solitons:

$$
\psi_{6}=\sqrt{2} \frac{G_{6}}{F_{6}}, \quad u_{6}=\left(2 \ln F_{6}\right)_{x x}
$$

with

$$
\begin{aligned}
F_{6} & =\sum \exp \left[\sum_{j=1}^{6} \mu_{j} \eta_{j}+\sum_{j<l}^{6} \mu_{j} \mu_{l} \ln A_{j l}\right], \\
G_{6} & =\sum \exp \left[\sum_{j=1}^{6} \mu_{j} c_{j} \eta_{j}+\sum_{j<l}^{6} \mu_{j} \mu_{l} \ln A_{j l}\right],
\end{aligned}
$$

where the $\operatorname{sign} \Sigma$ written before $\exp$ means that every $\mu_{j}, j=1,2, \cdots, 6$ assigns the values 0 or 1 . Moreover, $\eta_{j}$ and $A_{j l}$ in (62) are given by

$$
\begin{aligned}
\eta_{j} & =k_{j} x+p_{j} y-\omega_{j} t+\eta_{j}^{0}, \quad j=1,2, \cdots, N, \\
A_{j l} & =\frac{k_{j} k_{l}\left(k_{j}-k_{l}\right)^{2}+\left(p_{l} k_{j}-p_{j} k_{l}\right)^{2}}{k_{j} k_{l}\left(k_{j}+k_{l}\right)^{2}+\left(p_{l} k_{j}-p_{j} k_{l}\right)^{2}}, \quad c_{j}=\frac{i p_{j}+k_{j}^{2}}{i p_{j}-k_{j}^{2}},
\end{aligned}
$$

and the parameters $p_{j}, k_{j}, \omega_{j}$ are meeting the dispersion relation:

$$
\omega_{j}=\frac{\left(k_{j}^{4}-3 p_{j}^{2}\right)\left(k_{j}^{4}+p_{j}^{2}\right)-4 \kappa k_{j}^{4}}{\left(k_{j}^{4}+p_{j}^{2}\right) k_{j}} .
$$


Here $p_{1,2}, k_{1,2}, \omega_{1,2}, \eta_{1,2}^{0}$ further satisfy the parameter constraints in Eq. (87) while $p_{j}, k_{j}, \omega_{j}, \eta_{j}^{0}, j=3,4,5,6$ are real. Under this setting, we observe the fact that

$$
\omega_{1}=\omega_{2}^{*}, \quad c_{1} c_{2}^{*}=1, \quad A_{1 j}=A_{2 j}^{*}, j=3,4,5,6 .
$$

Therefore, we could use a group of variable transformations:

$$
\begin{aligned}
& \eta_{1}=\eta_{1 R}+\eta_{1 I} i, \quad A_{1 j}=\rho_{1 j} e^{i \phi_{1 j}}, j=3,4,5,6, \\
& \eta_{2}=\eta_{1 R}-\eta_{1 I} i, \quad c_{1}=\rho_{c_{1}} e^{i \phi_{c_{1}}}, \quad c_{2}=\frac{1}{\rho_{c_{1}}} e^{i \phi_{c_{1}}},
\end{aligned}
$$

where $\eta_{1 R}, \eta_{1 I}, \rho_{c_{1}}, \phi_{c_{1}}, \rho_{1 j}, \phi_{1 j}, j=3,4,5,6$ are real. Figure 9 shows a typical ellastic collision of a breather and four dark line solitons.

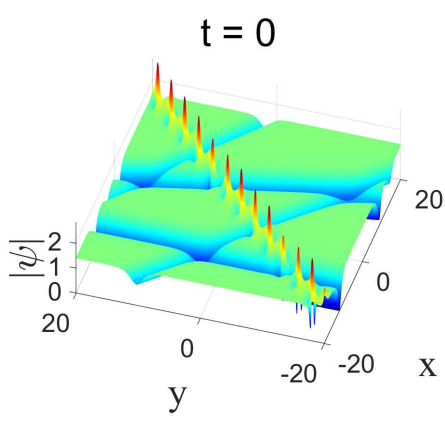

(a)

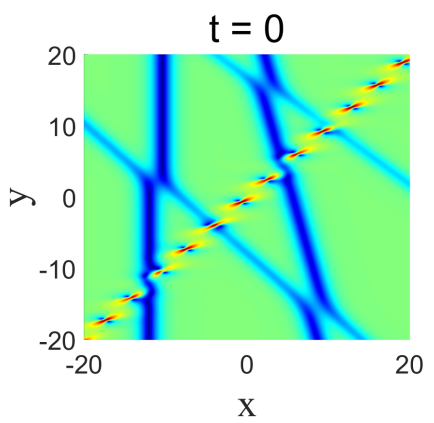

(b)

Fig. 9 The graphs demonstrating the elastic collision among a breather and four dark line solitons with the following choice of the parameters: $\eta_{1}^{0}=0, \eta_{2}^{0}=0, \eta_{3}^{0}=13, \eta_{4}^{0}=-20$, $\eta_{5}^{0}=16, \eta_{6}^{0}=0, \lambda_{11}=1, \lambda_{12}=0, a_{1}=-\frac{1}{2}, b_{1}=-1, q_{11}=\frac{2}{3}, q_{12}=-\frac{1}{2}, q_{21}=\frac{2}{3}, q_{22}=-\frac{3}{5}$, $q_{31}=\frac{2}{3}, q_{32}=0, q_{41}=\frac{2}{3}, q_{42}=-\frac{1}{8}$.

Again, for the convenience of solving the constraint condition of resonant collision, we use the following variable transformations:

$$
\begin{array}{ll}
k_{1}=\lambda_{11}+\lambda_{12} i, & p_{1}=2\left(\lambda_{11}+\lambda_{12} i\right)\left(a_{1}+b_{1} i\right), \\
k_{2}=\lambda_{11}-\lambda_{12} i, & p_{2}=2\left(\lambda_{11}-\lambda_{12} i\right)\left(a_{1}-b_{1} i\right), \\
k_{j}=2 q_{j 1}, & p_{j}=-4 q_{j 1} q_{j 2}, j=3,4,5,6 .
\end{array}
$$

Under these transformations, $A_{1 j}, j=3,4,5,6$ could be rewritten as

$$
A_{1 j}=\frac{N_{1 j} N_{2 j}}{D_{1 j} D_{2 j}}
$$

where

$$
\begin{aligned}
& N_{1 j}=\left(2 q_{j 2}+\lambda_{12}+2 a_{1}\right) i+\lambda_{11}-2 q_{j 1}-2 b_{1}, \\
& N_{2 j}=\left(2 q_{j 2}-\lambda_{12}+2 a_{1}\right) i-\lambda_{11}+2 q_{j 1}-2 b_{1}, \\
& D_{1 j}=\left(2 q_{j 2}+\lambda_{12}+2 a_{1}\right) i+\lambda_{11}+2 q_{j 1}-2 b_{1}, \\
& D_{2 j}=\left(2 q_{j 2}-\lambda_{12}+2 a_{1}\right) i-\lambda_{11}-2 q_{j 1}-2 b_{1} .
\end{aligned}
$$


The condition that the breather along $\eta_{1 R}$ resonantly collides with solitons along $\eta_{j}, j=3,4,5,6$ is that the phase shift goes to infinity. We have obtained in (13) that $\Delta \Phi \propto \ln A_{1 j}$, which implies that $A_{1 j}=0$ or $A_{1 j} \rightarrow \infty$. Observing the structure of $A_{1 j}$ in (68), we realize that there are four possible ways to make $\ln A_{1 j}$ infinite $\left(N_{1 j}=0, N_{2 j}=0, D_{1 j} \rightarrow \infty, D_{2 j} \rightarrow \infty\right)$. According to these results, let us set $A_{13}, A_{14}=0$ and $A_{15}, A_{16} \rightarrow \infty$. Further, $N_{13}=0, N_{24}=0, D_{15}=0$, $D_{16}=0$, and thus we get the following constraint conditions:

$$
\begin{aligned}
& \left\{\begin{array}{l}
q_{31}=\frac{1}{2} \lambda_{11}-b_{1} \\
q_{32}=-\frac{1}{2} \lambda_{12}-a_{1}
\end{array},,\left\{\begin{array}{l}
q_{41}=\frac{1}{2} \lambda_{11}+b_{1} \\
q_{42}=\frac{1}{2} \lambda_{12}-a_{1}
\end{array},\right.\right. \\
& \left\{\begin{array}{l}
q_{51}=-\frac{1}{2} \lambda_{11}-b_{1} \\
q_{52}=\frac{1}{2} \lambda_{12}-a_{1}
\end{array},\left\{\begin{array}{l}
q_{61}=-\frac{1}{2} \lambda_{11}+b_{1} \\
q_{62}=-\frac{1}{2} \lambda_{12}-a_{1}
\end{array} .\right.\right.
\end{aligned}
$$

Under these conditions, some extra constraints appear, which are as follows

$$
\eta_{3}+\eta_{6}=\eta_{3}^{0}+\eta_{6}^{0}, \quad \eta_{4}+\eta_{5}=\eta_{4}^{0}+\eta_{5}^{0}, \quad c_{3} c_{6}=1, \quad c_{4} c_{5}=1, \quad A_{34}=A_{56} .
$$

These constraints reveal that once the breather resonantly collides with four dark line solitons, degeneration of other two dark line solitons will happen. In other words, two dark line solitons in Figure 9 will disappear. We introduce the constraints (70) into the solution (61), and consider the extra constraints in (71). Thus we obtain the solution describing the resonant collision among a breather and four dark line solitons:

$$
\psi_{6 r}=\sqrt{2} \frac{G_{6 r}}{F_{6 r}}, \quad u_{6 r}=\left(2 \ln F_{6 r}\right)_{x x},
$$

where

$$
\begin{aligned}
F_{6 r} & =f_{61}+A_{12} e^{2 \eta_{1 R}} f_{62}+2 e^{\eta_{1 R}} \cos \eta_{1 I} \\
G_{6 r} & =g_{61}+A_{12} e^{2 \eta_{1 R}+2 i \phi_{c_{1}}} g_{62}+2 e^{\eta_{1 R}+i \phi_{c_{1}}} \cosh \left(\eta_{1 I} i+\ln \rho_{c_{1}}\right)
\end{aligned}
$$

with

$$
\begin{aligned}
f_{61}= & 1+e^{\eta_{3}^{0}+\eta_{6}^{0}}\left(e^{-\eta_{6}}+A_{56}\right)+e^{\eta_{4}^{0}+\eta_{5}^{0}}\left(e^{-\eta_{5}}+A_{56}\right) \\
& +A_{56} e^{\eta_{3}^{0}+\eta_{4}^{0}+\eta_{5}^{0}+\eta_{6}^{0}}\left(A_{56}+e^{-\eta_{5}}+e^{-\eta_{6}}+e^{-\eta_{5}-\eta_{6}}\right), \\
f_{62}= & 1+e^{\eta_{5}}+e^{\eta_{6}}+A_{56} e^{\eta_{5}+\eta_{6}}, \\
g_{61}= & 1+e^{\eta_{3}^{0}+\eta_{6}^{0}}\left(\frac{1}{c_{6}} e^{-\eta_{6}}+A_{56}\right)+e^{\eta_{4}^{0}+\eta_{5}^{0}}\left(\frac{1}{c_{5}} e^{-\eta_{5}}+A_{56}\right) \\
& +A_{56} e^{\eta_{3}^{0}+\eta_{4}^{0}+\eta_{5}^{0}+\eta_{6}^{0}}\left(A_{56}+\frac{1}{c_{5}} e^{-\eta_{5}}+\frac{1}{c_{6}} e^{-\eta_{6}}+\frac{1}{c_{5} c_{6}} e^{-\eta_{5}-\eta_{6}}\right), \\
g_{62}= & 1+c_{5} e^{\eta_{5}}+c_{6} e^{\eta_{6}}+A_{56} c_{5} c_{6} e^{\eta_{5}+\eta_{6}} .
\end{aligned}
$$

According to the trajectories on which the breather and the dark line solitons are moving along, the resonant collisions are classified into two types:

(1) Obliquely resonant collision of four dark line solitons and a breather when $\frac{k_{1 R}}{p_{1 R}} \neq \frac{k_{j}}{p_{j}}, j=3,4,5,6$. Thus, when the condition $\frac{k_{1 R}}{p_{1 R}} \neq \frac{k_{j}}{p_{j}}, j=3,4,5,6$ holds, the breather will obliquely intersect with the dark line soliton in the $(x, y)$ plane. 
Before collision:

The dark line soliton $\left(\eta_{5} \approx 0, \eta_{6} \rightarrow-\infty, \eta_{1 R} \rightarrow-\infty\right)$ :

$$
\psi_{\mathbf{S}}^{5-} \approx \sqrt{2} \frac{1+\left(\frac{e^{-\eta_{4}^{0}-\eta_{5}^{0}}}{A_{56}}+1\right) c_{5} e^{\eta_{5}}}{1+\left(\frac{e^{-\eta_{4}^{0}-\eta_{5}^{0}}}{A_{56}}+1\right) e^{\eta_{5}}} ;
$$

The dark line soliton $\left(\eta_{6} \approx 0, \eta_{5} \rightarrow-\infty, \eta_{1 R} \rightarrow-\infty\right)$ :

$$
\psi_{\mathbf{S}}^{6-} \approx \sqrt{2} \frac{1+\left(\frac{e^{-\eta_{3}^{0}-\eta_{6}^{0}}}{A_{56}}+1\right) c_{6} e^{\eta_{6}}}{1+\left(\frac{e^{-\eta_{3}^{0}-\eta_{6}^{0}}}{A_{56}}+1\right) e^{\eta_{6}}} ;
$$

The breather $\left(\eta_{1 R} \approx 0, \eta_{5} \rightarrow+\infty, \eta_{6} \rightarrow+\infty\right)$ :

$$
\psi_{\mathbf{B}}^{+} \approx \sqrt{2} c_{5} c_{6} ;
$$

After collision:

The dark line soliton $\left(\eta_{5} \approx 0, \eta_{6} \rightarrow+\infty, \eta_{1 R} \rightarrow+\infty\right)$ :

$$
\psi_{\mathbf{S}}^{5+} \approx \sqrt{2} \frac{1+A_{56} c_{5} e^{\eta_{5}}}{1+A_{56} e^{\eta_{5}}} ;
$$

The dark line soliton $\left(\eta_{6} \approx 0, \eta_{5} \rightarrow+\infty, \eta_{1 R} \rightarrow+\infty\right)$ :

$$
\psi_{\mathbf{S}}^{6+} \approx \sqrt{2} \frac{1+A_{56} c_{6} e^{\eta_{6}}}{1+A_{56} e^{\eta_{6}}} ;
$$

The breather $\left(\eta_{1 R} \approx 0, \eta_{5} \rightarrow-\infty, \eta_{6} \rightarrow-\infty\right)$ :

$$
\psi_{\mathbf{B}}^{-} \approx \sqrt{2} c_{5} c_{6}
$$

By making an analysis of the above written expressions, and by comparing the equations (75) and (76) with the equations (78) and (79), then

$$
\Delta \Phi_{\mathbf{S}}^{5}=\ln \left(\frac{e^{-\eta_{4}^{0}-\eta_{5}^{0}}+A_{56}}{A_{56}^{2}}\right), \quad \Delta \Phi_{\mathbf{S}}^{6}=\ln \left(\frac{e^{-\eta_{3}^{0}-\eta_{6}^{0}}+A_{56}}{A_{56}^{2}}\right),
$$

where $\Delta \Phi_{\mathrm{S}}^{5}$ and $\Delta \Phi_{\mathrm{S}}^{6}$ represent the phase shifts of dark line solitons along $\eta_{5} \approx 0$ and $\eta_{6} \approx 0$, respectively. Notice that $\eta_{3}^{0}$ and $\eta_{4}^{0}$ are independent of $\eta_{5}$ and $\eta_{6}$, which means that the phase shift could be set by changing $\eta_{3}^{0}$ and $\eta_{4}^{0}$ directly and thus has no impact on the amplitudes, shapes, and velocities of solitons. Figure 10 exhibits the evolution of the solution describing the oblique resonant collision among a breather and four solitons, where we can observe that there is a periodic waveform situated between the two dark line solitons, seperating them at a long enough time before and after collision. Note that other two dark line solitons are disappeared because of the occurrence of resonance. The original four dark line solitons are shown in Figure 9 without resonance. Similar to subsection 3.1, Figure 


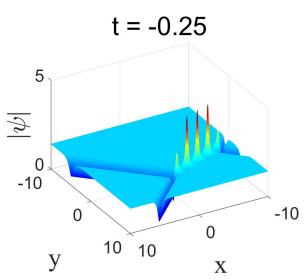

(a)

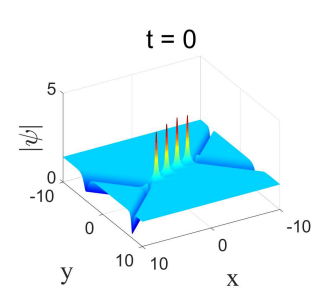

(b)

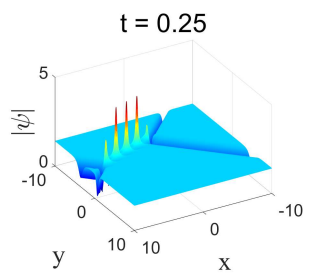

(c)

Fig. 10 The graphs of evolution of the solution describing the oblique resonant collision among a breather and four solitons with the following set of parameters: $\eta_{1}^{0}=0, \eta_{2}^{0}=0, \eta_{3}^{0}=-15$, $\eta_{4}^{0}=-15, \eta_{5}^{0}=-15, \eta_{6}^{0}=-15, \lambda_{11}=\frac{3}{2}, \lambda_{12}=1, a_{1}=0, b_{1}=2$.

10 also shows that the breather is remarkably localized in space and is not localized in time due to the the resonance.

(2) Parallel resonant collision of four dark line solitons and a breather when $\frac{k_{1 R}}{p_{1 R}}=\frac{k_{j}}{p_{j}}, j=3,4,5,6$. When the condition $\frac{k_{1 R}}{p_{1 R}}=\frac{k_{j}}{p_{j}}, j=3,4,5,6$ holds, the breather and the dark line soliton are parallel in the $(x, y)$ plane. Furthermore, if we analyze the previous result given in Eq. (25), we obtain that the constraint condition of parallel resonant collision is $\lambda_{12}=0$. Thus the solution (72) with parameters $q_{j 1}, q_{j 2}, j=3,4,5,6$ given by (70) and $\lambda_{12}=0$ illustrates the parallel resonant collision among a breather and four dark line solitons. Figure 11 exhibits the corresponding evolution dynamics; we see that in this case the whole dynamical process almost is very similar to what resonant collision among a breather and two dark line solitons displays, a result that is in complete accordance to our analysis in Type (2) at subsection 3.1. Similarly, the breather in Figure 10 is completely localized in time and is not localized in space.

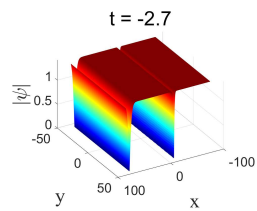

(a)

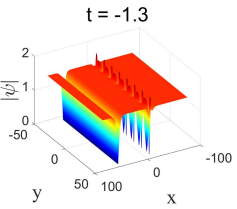

(b)

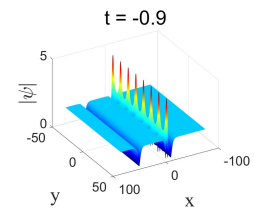

(c)

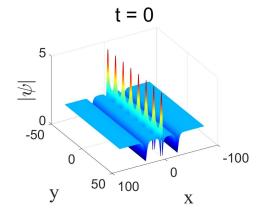

(d)

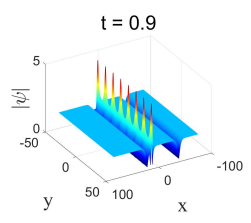

(e)

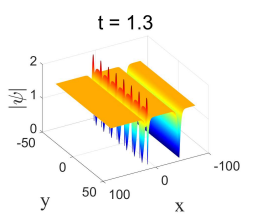

(f)

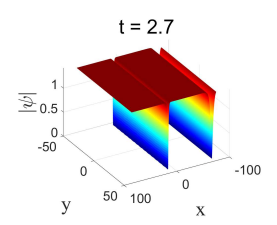

(g)

Fig. 11 The graphs of evolution of parallel resonant collision among a breather and four dark line solitons with the following choice of the parameters: $\eta_{1}^{0}=0, \eta_{2}^{0}=0, \eta_{3}^{0}=-15, \eta_{4}^{0}=-55$, $\eta_{5}^{0}=-55, \eta_{6}^{0}=-15, \lambda_{11}=\frac{1}{2}, \lambda_{12}=0, a_{1}=0, b_{1}=-\frac{1}{2}$. 


\section{Summary and discussion}

In this paper, we have used the Hirota bilinear method to solve the Mel'nikov equation (1). Using this powerful method, several combinations of various types of waveforms, including solitons, breathers, and lumps, have been obtained. We have found that the elastic collisions among different waveforms can be transformed into resonant collisions when the phase shifts of the involved solitary waves go to infinity. Both parallel and obliquely resonant collisions between different types of solitary waves have been investigated. The resonant collision of a lump and a dark line soliton has been obtained as the limit case of the resonant collision of a breather and a dark line soliton. It shows the fusing process of the lump into the dark line soliton. Then, we have pointed out that the resonant collision of a lump and two dark line solitons is also obtained as the limit case of the resonant collision among a breather and two dark line solitons; in this case, first, the lump is ejected from a dark line soliton (that is, via a fission process) and then the lump is fusing into the other dark line soliton. We have studied in detail the resonant collision scennarios between a breather and a dark line soliton and between a breather and two dark line solitons. Moreover, there is a unique phenomenon have been investigated that when a resonant collision among a breather and four dark line solitons occurs, an interesting situation occurs, namely two of the four dark line solitons are degenerate and the corresponding solution displays the same shape as that of the resonant collision among a breather and two dark line solitons, except for the phase shifts of the solitons, which are independent of the parameters controlling the waveforms of the solitons and the breather. The analysis developed in this work can be applied to the study of the interaction scenarios and of the resonant collisions between waveforms that are obtained as exact solutions of other nonlinear dynamical systems with applications in diverse physics and engineering settings.

Funding The work was supported by the National Natural Science Foundation of China under Grant No. 12071304.

Conflict statement We declare we have no conflict of interests.

Ethical statement Authors declare that they comply with ethical standards.

Data availability statement Data sharing not applicable to this article as no datasets were generated or analysed during the current study.

\section{Appendix}

In this Appendix, we present the mixed solutions consisting of dark line solitons and breathers to the Mel'nikov equation (1), which are constructed by using the Hirota's direct method [3].

Under the variable transformation

$$
=\sqrt{2} \frac{G}{F}, \quad u=(2 \ln F)_{x x},
$$


where $F$ and $G$ are real and complex functions of $x, y, t$, respectively, the Mel'nikov equation (1) is casted into the following set of bilinear equations

$$
\begin{aligned}
\left(3 D_{y}^{2}-D_{x} D_{t}-D_{x}^{4}\right) F \cdot F & =2 \kappa\left(F^{2}-G G^{*}\right) \\
\left(D_{x}^{2}-i D_{y}\right) G \cdot F & =0
\end{aligned}
$$

where $D$ is Hirota's operator [3].

The Mel'nikov equation (1) admits the following solutions

$$
\psi_{N}=\sqrt{2} \frac{G_{N}}{F_{N}}, \quad u_{N}=\left(2 \ln F_{N}\right)_{x x}
$$

with

$$
\begin{aligned}
F_{N} & =\sum \exp \left[\sum_{j=1}^{N} \mu_{j} \eta_{j}+\sum_{j<l}^{N} \mu_{j} \mu_{l} \ln A_{j l}\right], \\
G_{N} & =\sum \exp \left[\sum_{j=1}^{N} \mu_{j} c_{j} \eta_{j}+\sum_{j<l}^{N} \mu_{j} \mu_{l} \ln A_{j l}\right], \\
\eta_{j} & =k_{j} x+p_{j} y-\omega_{j} t+\eta_{j}^{0}, \quad j=1,2, \cdots, N \\
A_{j l} & =\frac{k_{j} k_{l}\left(k_{j}-k_{l}\right)^{2}+\left(p_{l} k_{j}-p_{j} k_{l}\right)^{2}}{k_{j} k_{l}\left(k_{j}+k_{l}\right)^{2}+\left(p_{l} k_{j}-p_{j} k_{l}\right)^{2}}, \quad c_{j}=\frac{i p_{j}+k_{j}^{2}}{i p_{j}-k_{j}^{2}},
\end{aligned}
$$

where $p_{j}, k_{j}$, and $\omega_{j}$ satisfy the dispersion relation

$$
\omega_{j} k_{j}\left(k_{j}^{4}+p_{j}^{2}\right)-\left(k_{j}^{4}-3 p_{j}^{2}\right)\left(k_{j}^{4}+p_{j}^{2}\right)+4 \kappa k_{j}^{4}=0 .
$$

For different conditions on the parameters, this solution corresponds to distinct types of solitary waves:

(a) The $N$ dark line solitons for real parameters $k_{j}, p_{j}, \omega_{j}, \eta_{j}^{0}(j=1,2, \cdots, N)$.

(b) The $\widehat{N}$ periodic solitons for complex parameters $k_{j}, p_{j}, \omega_{j}, \eta_{j}^{0}$ meeting the following relations involving the complex conjugation:

$$
N=2 \widehat{N}, k_{2 j}=k_{2 j-1}^{*}, p_{2 j}=p_{2 j-1}^{*}, \omega_{2 j}=\omega_{2 j-1}^{*}, \eta_{2 j}^{0}=\eta_{2 j-1}^{0 *}, j=1,2, \cdots, \widehat{N} .
$$

(c) The mixed solution consisting of $\widehat{N}$ periodic solitons and $(N-2 \widehat{N})$ dark line solitons for the complex parameters $k_{j}, p_{j}, \omega_{j}, \eta_{j}^{0}(j=1,2, \cdots 2 \widehat{N})$ obeying the relations involving the complex conjugation, which are given by Eq. (87), and the real parameters $k_{s}, p_{s}, \omega_{s}, \eta_{s}^{0}(s=\widehat{N}+1, \widehat{N}+2, \cdots, N)$.

\section{References}

1. Zabusky, N.J., Kruskal, M.D.: Interaction of solitons in a collisionless plasma and the recurrence of initial states. Phys. Rev. Lett. 15, 240-243 (1965)

2. Gardner, C. S., Greene, J. M., Kruskal, M. D., and Miura, R. M.: Method for solving the Korteweg-deVries equation, Phys. Rev. Lett. 19, 1095-1097(1967).

3. Hirota, R.: The Direct Method in Soliton Theory. Cambridge University Press, Cambridge (2004) 
4. Gardner, C. S., Greene, J. M., Kruskal, M. D., and Miura, R. M.: Korteweg-deVries Equation and Generalizations VI: Methods for Exact Solution, Comm. Pure Appl. Math. 27, 97-133(1974).

5. Airault, H., McKean, H.P., Moser, J.: Rational and elliptic solutions of the Korteweg-de Vries equation and a related many-body problem. Comm. Pure Appl. Math. 30, 95-148 (1977)

6. Adler, M., Moser, J.: On a class of polynomials connected with the Korteweg-de Vries equation. Commun. Math. Phys. 61, 1-30 (1978)

7. Ablowitz, M.J., Satsuma, J.: Solitons and rational solutions of nonlinear evolution equations. J. Math. Phys. 19, 2180-2186 (1978)

8. Satsuma, J., Ablowitz, M.J.: Two-dimensional lumps in nonlinear dispersive systems. J. Math. Phys. 20, 1496-1503 (1979)

9. Miles, J.W.: Resonantly interacting solitary waves. J. Fluid Mech. 79, 171-179 (1977)

10. Newell, A.C., Redekopp, L.G.: Breakdown of Zakharov-Shabat theory and soliton creation. Phys. Rev. Lett. 38, 377-380 (1977)

11. Kako F., Yajima, N.: Interaction of ion-acoustic solitons in two-dimensional space. J. Phys. Soc. Jpn. 49, 2063-2071(1980)

12. Folkes, P.A., Ikezi, H., Davis, R.: Two-dimensional interaction of ion-acoustic solitons. Phys. Rev. Lett. 45, 902-904 (1980)

13. Nishida, Y., Nagasawa, T.: Oblique collision of plane ion-acoustic solitons. Phys. Rev. Lett. 45, 1626-1629(1980)

14. Ohkuma, K., Wadati, M.: The Kadomtsev-Petviashvili equation: the trace method and the soliton resonances. J. Phys. Soc. Jpn. 52, 749-760 (1983)

15. Mel'nikov, V.K.: On equations for wave interactions. Lett. Math. Phys. 7, 129-136 (1983)

16. Mel'nikov, V.K.: Wave emission and absorption in a nonlinear integrable system. Phys. Lett. A 118, 22-24 (1986)

17. Mel'nikov, V.K.: Reflection of waves in nonlinear integrable systems. J. Math. Phys. 28, 2603-2609 (1987)

18. Mel'nikov, V.K.: A direct method for deriving a multi-soliton solution for the problem of interaction of waves on the x, y plane. Commum. Math. Phys. 112, 639-652 (1987)

19. Hase, Y., Hirota, R., Ohta, Y.: Soliton solutions of the Mel'nikov equations. J. Phys. Soc. Jpn. 58, 2713-2720 (1989)

20. Kumar, C.S., Radha, R., Lakshmanan, M.: Exponentially localized solutions of Mel'nikov equation. Chaos, Solitons and Fractals 22, 705-712 (2004)

21. $\mathrm{Mu}, \mathrm{G}$., Qin, Z.Y.: Two spatial dimensional N-rogue waves and their dynamics in Mel'nikov equation. Nonlinear Anal. RWA 18, 1-13 (2014)

22. Deng, Y., Jia, R., Lin, J.: Lump and mixed rogue-soliton solutions of the (2+1)-dimensional Mel'nikov system. Complexity 2019, 1420274 (2019)

23. Sun, B.N., Wazwaz, A.M.: Interaction of lumps and dark solitons in the Mel'nikov equation. Nonlinear Dyn. 94, 2841-2862 (2018)

24. Zhang, X.E., Chen, Y.: Hybrid solutions to Mel'nikov system. Nonlinear. Dyn. 92, 20492059 (2018)

25. Rao, J.G., Malomed, B.A., Cheng, Y., He, J.S.: Dynamics of Interaction between lumps and solitons in the Mel'nikov equation. Commun. Nonlinear Sci. Numer. Simulat. 91, 105429 (2020)

26. Liu, W., Meng, Y., Qiao, X.: Two-dimensional new coherent structures of lump-soliton solutions for the Mel'nikov equation. Accepted by Modern Phys. Lett. B (2021), see https://doi.org/10.1142/S0217984921504169

27. Xu, G.Q., Wazwaz, A.M.: Bidirectional solitons and interaction solutions for a new integrable fifth-order nonlinear equation with temporal and spatial dispersion. Nonlinear Dyn. 101, 581-595 (2020)

28. Ankiewicz, A., Bokaeeyan, M., Chang, W.: Understanding general rogue wave solutions of the Gardner equation. Rom. Rep. Phys. 72, 119 (2020)

29. Guo, L.J., Ward, C.B., Mylonas, I.K., Kevrekidis, P.G.: Solitary waves of the CamassaHolm derivative nonlinear Schrödinger equation. Rom. Rep. Phys. 72, 107 (2020)

30. Guo, J., He, J., Li, M., Mihalache, D.: Exact solutions with elastic interactions for the (2+1)-dimensional extended Kadomtsev-Petviashvili equation. Nonlinear Dyn. 101, 2413$2422(2020)$

31. Mihalache, D.: Localized structures in optical and matter-wave media: a selection of recent studies. Rom. Rep. Phys. 73, 403 (2021)

32. Ma, Y., Wazwaz, A.M., Li, B.Q.: New extended Kadomtsev-Petviashvili equation: multiple soliton solutions, breather, lump and interaction solutions. Nonlinear Dyn. 104, 1581-1594 (2021) 